\title{
Possibilidades para a mudança do modelo obstétrico hegemônico pelas enfermeiras obstétricas
}

\author{
Opportunities for nurse midwives to bring change to the hegemonic model of obstetrics \\ Posibilidades para el cambio del modelo obstétrico hegemónico por las enfermeras obstétricas
}

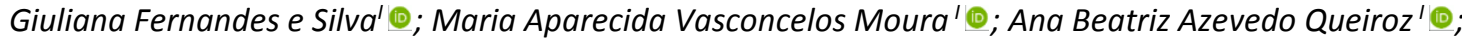

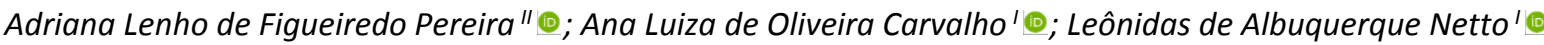 \\ 'Universidade Federal do Rio de Janeiro, Rio de Janeiro, RJ, Brasil; "Universidade do Estado do Rio de Janeiro, Rio de Janeiro, RJ, Brasil
}

\begin{abstract}
RESUMO
Objetivo: descrever o uso das tecnologias de cuidado da enfermeira obstétrica qualificada na modalidade de residência e sua relação com a práxis profissional. Método: estudo qualitativo com 13 enfermeiras obstétricas atuantes em duas maternidades públicas do Rio de Janeiro, Brasil. Dados coletados por entrevista individual, semiestruturada e análise hermenêutica-dialética. Resultados: a transição do modelo intervencionista estrutura-se no cuidado humanizado com a incorporação de um modelo centrado nas boas práticas e nas tecnologias não invasivas de cuidado da enfermeira obstétrica. A práxis da enfermeira obstétrica contribui para a transformação qualitativa deste cenário, com resgate sobre a fisiologia, o fortalecimento de vínculo e empoderamento da mulher, ressignificando o momento do parto. Conclusão: a prática da enfermeira e o uso das tecnologias não invasivas constituem possibilidades para ruptura do modelo hegemônico culturalmente instituído no Brasil, sendo necessária ainda a constituição de um consenso que supere o senso comum.
\end{abstract}

Descritores: Saúde da Mulher; Enfermeiras Obstétricas; Parto Normal; Hermenêutica.

\section{ABSTRACT}

Objective: to describe the use of care technologies by residency-qualified nurse midwives and their relationship with professional praxis. Method: in this qualitative study with 13 nurse midwives at two public maternity hospitals in Rio de Janeiro, Brazil, data were collected by individual, semi-structured interview and hermeneutic-dialectic analysis. Results: the transition from the interventionist model builds on humanized care by incorporating a model centered on nurse midwives' use of best practices and noninvasive care technologies. Their praxis contributes to qualitative change in this scenario by reinstating physiology, fostering stronger bonding, and empowering women, so as to re-signify the moment of childbirth. Conclusion: nurse midwives' praxis and use of noninvasive technologies constitute opportunities to break with the culturally established model hegemonic in Brazil, while a consensus still needs to be built to surmount common sense.

Descriptors: Women's Health; Nurse Midwives; Natural Childbirth; Hermeneutics.

\section{RESUMEN}

Objetivo: describir el uso de tecnologías asistenciales por parte de enfermeras parteras tituladas en residencia y su relación con la praxis profesional. Método: en este estudio cualitativo con 13 enfermeras parteras de dos maternidades públicas de Río de Janeiro, Brasil, los datos fueron recolectados mediante entrevista individual, semiestructurada y análisis hermenéuticodialéctico. Resultados: la transición del modelo intervencionista se basa en la atención humanizada al incorporar un modelo centrado en el uso de las mejores prácticas y tecnologías de atención no invasiva por parte de las enfermeras parteras. Su praxis contribuye al cambio cualitativo en este escenario al reinstaurar la fisiología, fomentar vínculos más fuertes y empoderar a las mujeres, para resignificar el momento del parto. Conclusión: la praxis de las enfermeras parteras y el uso de tecnologías no invasivas constituyen oportunidades para romper con el modelo hegemónico culturalmente establecido en Brasil, mientras que aún debe construirse un consenso para superar el sentido común.

Descriptores: Salud de la Mujer; Enfermeras Obstetrices; Parto Normal; Hermenéutica.

\section{INTRODUÇÃO}

$\mathrm{Na}$ realidade dos serviços de saúde, visualiza-se a fragmentação da assistência onde medicalização e a institucionalização do parto tornaram o corpo da mulher objeto de intervenções, rompendo com o cuidado centrado no ambiente doméstico; estas eram mantidas relações afetivas, de solidariedade, segurança e confiança ${ }^{1,2}$.

Observa-se que a cultura assistencial hegemônica e o poder de influência da corporação médica diminuem as possibilidades de expansão de uma modalidade de assistência que modificando este padrão decisório no cenário hospitalocêntrico. Esta cultura decorre de uma sociedade tecnocrática que se organiza a partir de uma ideologia de progresso tecnológico, associada a concepções ou valores dessa população ${ }^{3}$.

Agradecimento à Coordenação de Aperfeiçoamento de Pessoal de Nível Superior (CAPES) pela bolsa de doutorado no Brasil e doutorado sanduíche na Espanha Autora correspondente: Giuliana Fernandes e Silva. E-mail: giulianafernandes@hotmail.com

Editora responsável: Helena Maria Scherlowski Leal David 
Este paradigma influencia a atenção ao parto e nascimento nos dias atuais e pauta-se no modelo de atenção obstétrica, favorecendo o uso indiscriminado de tecnologias, intervenções inadequadas e invasivas ao corpo da mulher. A prática da cesárea de rotina é violação dos direitos da parturiente refletindo na manutenção da taxa elevada de mortalidade materna ${ }^{3}$. O excesso de intervenções obstétricas na assistência hospitalar deixou de considerar os aspectos emocionais, culturais e humanos envolvidos neste processo, que se reveste de um caráter particular e vai além do processo de parir e nascer ${ }^{4}$. A predominância da medicalização do parto persiste, apesar da argumentação sobre a humanização em programas e políticas públicas, o que impede a integralidade da assistência humanizada ${ }^{5}$.

Houve a necessidade de estruturar o sistema de atenção com o intuito de atenuar o número de cesáreas e aprimorar a assistência obstétrica no Sistema Único de Saúde (SUS). Esta assistência deve abarcar as diretrizes políticas em uma rede de cuidados visando assegurar à mulher atenção humanizada, respeito à fisiologia do parto, detecção precoce de intercorrências gestacionais e acolhimento nas maternidades. $\mathrm{O}$ atendimento em Centro de Parto Normal por equipes multiprofissionais com a inserção e valorização da atuação da enfermeira obstétrica deve ser baseada em evidências científicas.

As instituições políticas e educacionais associadas à atuação das enfermeiras com sua práxis humanizada contribuem para a mudança do modelo vigente de atenção ao parto denominado tecnocrático, priorizando a intervenção da assistência, o uso abusivo de tecnologia invasiva, a passividade das mulheres durante o trabalho de parto, alterando o ritmo natural, impedindo a mulher de exercer seu protagonismo no parto e adotar práticas sem evidências científicas.

No intuito de superar esta cultura assistencial hegemônica e estabelecer uma nova cultura norteada sob o paradigma da humanização ao parto normal procura-se estimular a formação e o aprimoramento da enfermeira obstétrica, para que atuem de forma ativa, na mudança do modelo obstétrico.

Questiona-se com que finalidade as enfermeiras obstétricas utilizam as tecnologias de cuidado como intervenção no parto e nascimento. Considerando as relações de hegemonia culturalmente instituídas na atenção ao parto delineouse o objetivo de descrever o uso das tecnologias de cuidado pela enfermeira obstétrica qualificada na modalidade de residência e sua relação com a práxis profissional.

\section{MÉTODO}

Estudo descritivo, exploratório e de abordagem qualitativa ${ }^{6}$, norteado pelo Consolidated criteria for reporting qualitative research (COREQ) ${ }^{7}$. Participaram 13 enfermeiras obstétricas egressas de programas de residência obstétrica atuantes em maternidades públicas do município do Rio de Janeiro, Brasil.

A investigação qualitativa se ocupa do nível subjetivo e relacional da realidade social e é tratada através da história, do universo, significados, motivos, crenças, valores e das atitudes dos atores sociais. Procura compreender e interpretar de forma fidedigna a lógica interna dos participantes e oferece conhecimento de sua 'verdade'. As diferenças de interpretações frequentemente refletem uma compreensão multifacetada de fenômenos sociais $\operatorname{complexos}^{6}$.

A opção pelas participantes justifica-se pela necessidade de analisar a atuação dessas profissionais formadas nestes programas, instituídos em 2012, como estratégia do Ministério da Saúde para incentivo à formação de enfermeiras obstétricas no Programa Nacional de Residência em Enfermagem Obstétrica (PRONAENF). Este objetiva a formação de especialistas na residência direcionadas ao cuidado à saúde da mulher orientada pelas políticas de saúde vigentes no SUS. A escolha dos cenários ocorreu pela inserção e atuação profissional das enfermeiras na assistência ao parto de risco habitual e o fato deste constituir campo prático de formação para os cursos de residência.

O universo de profissionais elegíveis considerou que uma das maternidades apresentava 20 profissionais, distribuídas nos setores de acolhimento e sala de parto, das quais nove eram egressas do curso de residência. Na outra, dez profissionais atuavam na sala de parto e possuíam vínculo direto com a instituição; destas, quatro eram egressas do curso de residência. Totalizou-se 13 participantes da pesquisa. Os critérios de inclusão foram enfermeiras obstétricas egressas do curso de residência formadas após a criação e incentivo do PRONAENF, que atuavam como servidoras estatutárias ou trabalhadoras celetistas. Excluíram-se as enfermeiras que exerciam atividade de chefia de unidade e/ou estavam em situação de afastamento por licença ou férias.

No percurso da investigação, utilizou-se entrevista semiestruturada, contendo questões abertas e fechadas, referentes à caracterização do perfil socioprofissional, prática assistencial e o uso das tecnologias na assistência ao parto. As entrevistas ocorreram no período de outubro de 2016 a março de 2017 com duração média de 45 minutos, em horário de disponibilidade das participantes e realizadas em uma única vez, após convite às enfermeiras pela pesquisadora. Os depoimentos foram gravados em $\mathrm{mp} 4$, armazenados e, posteriormente, transcritos na íntegra para iniciar o tratamento de análise dos resultados. 
A produção do material ocorreu pela análise hermenêutica-dialética que realiza a síntese dos processos compreensivos e críticos em pesquisas qualitativas e analisa a comunicação da vida cotidiana e do senso comum. $\mathrm{A}$ hermenêutica se ocupa da arte de compreender textos, busca a compreensão e a interpretação; enquanto o método dialético introduz o princípio do conflito e da contradição como constitutivos da realidade e essenciais para a compreensão analítica6 .

O fluxograma de análise metodológica constituiu-se pela organização dos dados estabelecendo a identificação do material empírico e a classificação dos dados, detectando as ideias centrais e realizando a leitura de cada corpus de comunicação ${ }^{6}$. Em seguida, confrontaram-se os diferentes grupos e os dados foram construídos a partir dos pressupostos teóricos da práxis.

Protocolo de pesquisa aprovado pelos Comitês de Ética da Escola das instituições envolvidas, sob os Certificados

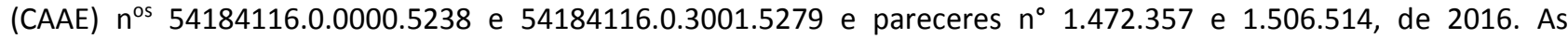
participantes receberam codificação alfanumérica (E1, E2... E13) para preservar o sigilo, anonimato, confiabilidade e garantia de participação voluntária, mediante assinatura do Termo de Consentimento Livre e Esclarecido.

\section{RESULTADOS E DISCUSSÃo}

As participantes apresentaram perfil condizente com o período de maior produtividade profissional. Todas eram mulheres com idade entre 26 e 36 anos e a maioria era casada. Relataram inserção no mercado de trabalho, logo após o término da residência, atuando na assistência ao parto normal. Verificou-se o investimento em sua formação na realização de cursos de aperfeiçoamento, capacitação sobre o parto e nascimento, amamentação, especialização em neonatologia, reanimação neonatal e em obstetrícia. Ressalta-se nos resultados uma participação dos profissionais na qualificação uma vez ao ano, o que representa uma busca pelo conhecimento atualizado na área obstétrica.

Ao analisar o depoimento das enfermeiras obstétricas observa-se a consonância de seus relatos no sentido de contribuir para a redução das intervenções à parturiente. Todas as entrevistadas citaram as principais tecnologias não invasivas de cuidado utilizadas, tais como a bola, o banho morno, balanço pélvico, aromaterapia, musicoterapia, penumbra, massagem, deambulação, acupressão, técnica do rebozo, banqueta e o uso de banheira. Esta prática consolida sua práxis profissional e firma possibilidades de mudança no cenário obstétrico, ainda que se vislumbrem disputas neste campo. A despeito destes conflitos, as enfermeiras empregam os recursos disponíveis nas instituições, algumas aproveitam as tecnologias de cuidado, as mais acessíveis para assistência ou o conhecimento que possuem, conforme as expressões a seguir.

Apesar das dificuldades, utilizamos a bola, cavalinho, aromaterapia, massagem, água e acupressão. O próprio ouvir, escutar, também é uma tecnologia, porque às vezes, a mulher só quer se expressar. O rebozo não tem aqui, mas trago ou às vezes, tento usar o lençol. (E11)

Quando conseguimos usamos a bola, o banho, deambulação, cavalinho, sais na água aquecida e banheira. Têm profissionais que trazem o rádio portátil e a gente utiliza a musicoterapia. (E13)

Assim que entra no box oferecemos o banho, porque elas já sabem pela visita da Rede Cegonha e pedem. No puerpério relatam que o banho ajudou. (E2)

A escuta ativa representa um momento de compartilhamento e tornar-se acessível as suas demandas com a parturiente; oportunidade também de uma tecnologia na atenção ao parto pelas enfermeiras ${ }^{8}$. Este compromisso de disponibilizar-se na entrega ao outro torna a assistência uma experiência particular, como fonte de expansão, possibilitando mudar o modelo obstétrico de atenção. A concepção tecnológica de cuidado de enfermagem obstétrica como relacional é construída desde o acolhimento da parturiente, estabelecimento de vínculo na visita à maternidade até o momento de proporcionar o uso das tecnologias durante o trabalho de parto.

No entanto, a práxis das enfermeiras não se limita ao modelo de atenção menos intervencionista; pauta-se na humanização, em sua competência assistencialista de eleger a melhor indicação à parturiente, distanciando-se do modelo biomédico, com base nas recomendações da Organização Mundial da Saúde. As enfermeiras conscientes e seguras com as práticas preconizadas e incentivadas optam pela sua esfera de atividade, a fim de contribuir ativamente para a qualidade da atenção ao parto normal.

Portanto, a prática das enfermeiras não pode ser analisada de modo pragmático, como mera objetivação de ações programáticas recomendadas, mas como atividade conscientemente orientada, composta por uma totalidade teóricoprática indissociável, constituída por um lado ideal teórico e um lado material propriamente prático ${ }^{9}$. Por isto, não podem ser isolados um do outro, o que remete à análise da práxis destas profissionais; pois não se trata de modo operacionalizado, mas compreende a atribuição de acolher e efetivar a dimensão subjetiva e relacional do cuidado à mulher, em todos os espaços de atenção ao parto. As enfermeiras também descreveram a contribuição das práticas e tecnologias utilizadas no parto normal frente às estratégias políticas de assistência ao parto: 
As tecnologias contribuem para evitar práticas intervencionistas desnecessárias e ajudar a humanização do parto, deixá-lo mais natural. Temos que intervir quando tem necessidade e deixar fluir naturalmente quando não tem. Instituir estas tecnologias é minimizar o índice de episiotomia e cesarianas melhorando a qualidade do parto. (E1)

Nossa prática contribui para ajudar a mulher. As tecnologias são para o alívio da dor e desconforto no trabalho de parto. Então, a diminuição de cesarianas e desfechos negativos é consequência do trabalho com nossas técnicas e mudanças no cenário obstétrico. (E2)

As políticas públicas de atenção à saúde da população feminina e as instituições de ensino profissional apontam a necessidade premente de aproximação efetiva e (re)significação da formação de recursos humanos à saúde. Seguindo a lógica da proposta de organização da atenção à saúde no Brasil observou-se na assistência obstétrica, o movimento para a mudança do modelo hegemônico de atenção biomédica e intervencionista para o modelo com menor grau de intervenção no parto e respeito à sua fisiologia.

Este movimento é fortemente notado na formação das enfermeiras obstétricas que, desde a graduação, norteiamse pelas Diretrizes Curriculares Nacionais e os Princípios do SUS, contribuindo para que sejam profissionais humanistas, críticas e reflexivas $^{10}$. Nessa perspectiva, as enfermeiras referiram que as políticas públicas de saúde têm incentivado a mudança do modelo assistencial obstétrico e descreveram o papel que possuem neste movimento de transformação.

Fui formada na proposta de mudança do poder hegemônico, de sair do centro e trabalhar as extremidades, entender que a mulher e sua família são mais importantes. (E4)

A enfermagem obstétrica está no modelo humanizado que há tempo já se discutia. Na residência sempre tivemos um olhar mais à frente, de fazer com que o modelo de atendimento fosse realmente focado na mulher, na família, nas necessidades e na coparticipação do processo de nascimento. Caminhamos para mudanças $e$ lutamos para fazer melhor o nosso espaço. (E13)

Frente à análise da práxis das enfermeiras a formação de profissionais que tenham pensamento crítico sobre a ruptura do modelo hegemônico constitui-se um grande desafio, pois está associada ao enfrentamento e à superação de paradigmas no cotidiano das instituições que buscam instituir mudanças e redefinir saberes e práticas. A articulação de um movimento que se empenha em mudar o modelo medicalizado e intervencionista deve caminhar para valores centrados na emancipação social das classes profissionais, na democracia efetiva dos direitos da mulher e no aproveitamento dos profissionais, sustentada em uma prática emancipatória, de sujeitos autônomos e protagonistas. Espera-se uma articulação com a luta política institucional por um Estado que governe com as organizações da sociedade civil pelas maiorias ${ }^{11}$, que atenda às necessidades e demandas das mulheres no parto e nascimento.

Ao resgatar o conceito de práxis como ação do homem sobre a matéria e criação de uma nova realidade ${ }^{9}$, as enfermeiras sedimentam suas atividades e seu conhecimento em uma práxis criadora (reflexiva), determinante qualitativamente produzindo uma nova realidade. Elas também ensejam a mudança do modelo de atenção obstétrica no Brasil, e acreditam que é possível a ruptura da hegemonia atual.

Desse modo, a práxis das enfermeiras obstétricas está sustentada sob o desenvolvimento das capacidades de pensar, produzir e de transformar a realidade. Esta ruptura segundo as participantes é a contribuição da enfermagem obstétrica para uma modelo transformador, em constante movimento que beneficie a humanização e a qualidade da assistência à saúde da mulher.

Na minha época, a assistência era medicalizada. As mulheres eram internadas com soro, ocitocina e dieta zero. Aqui começou outra proposta, colocamos um protocolo em que a enfermeira não ia acompanhar o trabalho de parto que fosse induzido com ocitocina; caso fosse colocado, o atendimento seria encaminhado ao médico; e como eles não querem ficar com todas as pacientes, atendiam ao nosso protocolo. (E5)

Nossa assistência é individualizada, incentivando o parto normal sem violência, explicando os benefícios de respeitar o corpo, a fisiologia e os benefícios ao bebê. Isso firma nossa assistência. Vejo que minha prática é diferente do modelo dominante. (E2)

As enfermeiras demonstram responsabilidade, ética, empenho e dedicação ao processo de modificação do cenário obstétrico e se percebem nas ações propostas pelas políticas públicas. Enfatizam que seu processo de trabalho está em conjunto com as iniciativas ministeriais para a redução das taxas de intervenção obstétrica e reconhecem a atividade concreta, com a qual se afirmam neste campo, uma vez que colaboram para desfechos positivos na condução do parto normal. Destacam, ainda, que sua assistência modifica a realidade e a luta para a mudança deve ser diária e todos os envolvidos devem incentivar a ruptura do modelo existente, sendo estes os esforços necessários para uma transformação efetiva.

As participantes descreveram a ligação entre o processo de rompimento da medicalização e tecnicismo e a sua práxis humanizadora revelando a luta no espaço de atenção ao parto normal hospitalar. Este movimento evidencia uma prática assistencial de cuidado em busca de reconhecimento emancipatório ${ }^{12}$. Evidencia-se em sua prática cotidiana na atenção 
ao parto, a construção decisória de romper com o modelo hegemônico, ao reconhecerem sua própria atuação nas maternidades, a autonomia em exercer sua prática, a relação com a equipe e com as estratégias das políticas públicas.

Nessa perspectiva, não há a intenção por parte da Enfermeira, de exercer domínio no campo, mas compartilhar conhecimentos quanto à melhor assistência e estabelecer parcerias que concorram à melhoria da qualidade da atenção à mulher.

Os depoimentos indicam uma fase transicional do modelo obstétrico, bem como mudanças na prática médica e na relação entre médicos e enfermeiras. Esta realidade revela a influência da práxis da enfermeira na atenção ao parto normal e a adesão aos procedimentos realizados como um processo dinâmico, multifatorial e comportamental resultante de um conjunto de determinantes que dependem de fatores subjetivos. É um momento de decisão mediado por traços da personalidade, nível cognitivo intelectual, crenças e contexto social do indivíduo. Assumir atitudes profissionais adequadas, com estímulo e conhecimento técnico-científico é aderir às práticas recomendadas de forma benéfica às mulheres assistidas no parto normal ${ }^{13}$.

Aqui as médicas não sabem para que serve a bola e perguntam: "Será que dá para usar? O que acha?" Outro dia tinha uma paciente com dilatação total, mas com o bebê alto e a médica falou comigo: "Está tão alto, o que você acha que tem que fazer?". Ela entende que isso é necessário, mas ainda não sabe utilizar esse conhecimento. (E11)

A atitude individual e coletiva, o comportamento e, principalmente, as competências das enfermeiras passaram a exercer influência na implementação de procedimentos e serviços nas maternidades diante da equipe médica. Ao mesmo tempo, a comunicação, a relação entre equipes e a facilidade de estar em uma instituição que respalda e incentiva um modelo de atenção distinto contribuem para a adoção de boas práticas de atenção ao parto normal. Todavia, essas mudanças possibilitam uma complexa convivência para a formação do profissional da saúde, permeados por distintos interesses e jogos de poder, no âmbito dos serviços de saúde ${ }^{14}$.

A assistência ao parto hospitalar realizada antes, prioritariamente por médicos, passou a ser compartilhada com enfermeiras obstétricas, profissionais que dominam o cuidado nesta área específica. Nasceu, neste momento, uma nova ciência do cuidado, focada na assistência perinatal humanizada. As tecnologias de cuidado foram desenvolvidas e aprimoradas no intuito de qualificar esta assistência e garantir a segurança do binômio no processo de parto e nascimento ${ }^{15}$. Neste pensamento, todo novo organismo histórico cria uma nova estrutura, cujos representantes especializados e portavozes só podem ser concebidos como os 'novos intelectuais', e não como a continuação da intelectualidade precedente. Se os novos intelectuais se colocam como continuação direta da precedente, não são verdadeiramente novos, mas conservadores do grupo historicamente superado ${ }^{16}$. As enfermeiras pela cientificidade ao longo da história e solidez tradicional da profissão conseguiram atingir o grau de desenvolvimento necessário para criar novas estruturas, porém ainda vivem no invólucro cultural da velha história, ou seja, imersas no modelo intervencionista e medicalizado.

Apesar de afirmarem que têm uma relação profissional respeitosa com a equipe médica percebe-se que há uma concorrência velada, em que as enfermeiras obstétricas procuram ganhar espaço e estabelecer seu poder, enquanto os médicos lutam para não perder o domínio do cenário obstétrico.

Sempre vai ter algum embate entre as duas profissões, porque são pessoas e formações diferentes. Em outras unidades, o embate é grande, só vai mudar quando mudar a formação acadêmica deles [médicos], porque é algo enraizado. A questão maior são os pediatras, que aspiram e manipulam os bebês, principalmente porque dizem que são poucos e não têm tempo para esperar o bebê fazer o contato pele a pele, nem esperar a saída da placenta ou parar de pulsar o cordão. (E7)

Mesmo que todo o processo do parto tenha sido assistido pela enfermeira obstétrica, com respeito à fisiologia e incentivo ao empoderamento da mulher, estas ainda precisam firmar seu espaço frente ao poder que os profissionais médicos exercem sobre elas no âmbito institucional. Embora tenham ganhado mais espaço na assistência por meio do uso de tecnologias de cuidado no parto para o alívio da dor, da administração da dieta, do incentivo ao contato pele a pele, das orientações para amamentação e primeiros cuidados do bebê ${ }^{17}$, persiste a resistência de outros profissionais na assistência em todo o processo do parto, dificultando sua emancipação neste cenário.

Não obstante, a maioria das enfermeiras mostrou que a experiência nestas maternidades, ainda que vivenciem alguns problemas decisórios é melhor, se comparada à realidade de outras instituições, onde não conseguem atuar com autonomia. Vale ressaltar que a disputa entre os profissionais só acarreta prejuízos às mulheres, pois causa um ambiente de tensões que não favorece nenhuma das categorias profissionais.

Todo grupo social que pretende conquistar sua hegemonia precisa passar por um processo constitutivo de sua identidade e intelectualidade, e por uma educação que exija a construção rigorosa de um saber mais avançado e socializado ${ }^{16}$. Evidenciar estes valores e analisar criticamente este percurso, de modo a entender o contexto contemporâneo obstétrico é um processo necessário que exige das enfermeiras a captação de instrumentos que propicie conhecimento, possibilitem novos saberes e promovam confiança em suas capacidades. 
Como limitação deste estudo têm-se o número reduzido de participantes mesmo abarcando todo o elenco de enfermeiras obstétricas egressas do Curso de Residência.

\section{CONCLUSÃO}

Nesse estudo, foi possível descrever o uso das tecnologias de cuidado utilizadas pela enfermeira obstétrica e sua relação com a práxis profissional. Estas enfermeiras conscientes de seu papel utilizaram sua experiência crítica para analisar e decidir como cuidar diante das mais diversas situações do cotidiano profissional, construindo um percurso de transformação para melhorar a qualidade da assistência ao parto normal hospitalar. A práxis da enfermeira obstétrica está elaborada sob uma visão global e sua prática se conforma com a política, a história e o conhecimento, considerados importantes na sua formação - atores críticos e ativos no cenário obstétrico.

Por fim, este estudo fortalece as evidências científicas sobre a inserção das enfermeiras obstétricas egressas da residência no que concerne ao incremento do uso das tecnologias de cuidado para alívio da dor e sua influência na atenção ao parto e nascimento. A formação da enfermeira obstétrica qualifica sua atuação no exercício profissional da prática humanizada, sendo necessária a integração interdisciplinar no cenário obstétrico.

\section{REFERÊNCIAS}

1. Betron ML, McClair TL, Currie S, Banerjee J. Expanding the agenda for addressing mistreatment in maternity care: a mapping review and gender analysis. Reprod. Health [Internet], 2018 [cited 2019 Jan 20]; 15(1):143. DOI: https://doi.org/10.1186/s12978-018-0584-6.

2. Benyamini Y, Molcho ML, Dan U, Gozlan M, Preis H. Women's attitudes towards the medicalization of childbirth and their associations with planned and actual modes of birth. Women Birth [Internet], 2017 [cited 2019 Jan 20]; 30(5):424-30. DOI: https://doi.org/10.1016/j.wombi.2017.03.007.

3. Rodrigues DP, Alves VH, Vieira RS, Leão DCMR, Paula ED, \& Pimentel MM. Obstetric violence in the context of labor and childbirth. J. Nurs. UFPE on line [Internet], 2018 [cited 2019 Jan 20]; 12(1):236-46. Available from: https://periodicos.ufpe.br/revistas/revistaenfermagem/article/view/23523.

4. Ministério da Saúde (Br). Secretaria de Ciência, Tecnologia e Insumos Estratégicos. Departamento de Gestão e Incorporação de Tecnologias em Saúde. Diretrizes nacionais de assistência ao parto normal: versão resumida [recurso eletrônico]. Brasília (DF): Ministério da Saúde; 2017. [cited 2019 Jan 20]. Available from

http://bvsms.saude.gov.br/bvs/publicacoes/diretrizes_nacionais_assistencia_parto_normal.pdf.

5. Giantaglia FN, Garcia ESGF, Rocha LCT da, et al. The care of nurses of an obstetric residence program under the scope of humanization. Rev. enferm. UFPE on line [Internet], 2017 [cited 2019 Jan 20]; 11(5):1882-90. Available from: https://periodicos.ufpe.br/revistas/revistaenfermagem/article/download/23337/18938.

6. Taquette SR, Minayo MC. An analysis of articles on qualitative studies conducted by doctors published in scientific journals in Brazil between 2004 and 2013. Physis [Internet], 2017 [cited 2019 Jan 20]; 27(2):357-74. DOI: http://dx.doi.org/10.1590/s010373312017000200010.

7. Tong A, Sainsbury P, Craig J. Consolidated criteria for reporting qualitative research (COREQ): a 32 -item checklist for interviews and focus groups. Int. J. Qual. Health Care [Internet], 2007 [cited 2019 Jan 20]; 19(6):349-57. DOI: https://doi.org/10.1093/intqhc/mzm042.

8. Alvares AS, Corrêa ÁCP, Nakagawa JTT, Teixeira RC, Nicolini AB, Medeiros RMK. Humanized practices of obstetric nurses: contributions in maternal welfare. Rev. Bras. Enferm. [Internet], 2018 [cited 2019 Jan 20]; 71(Suppl 6):2620-27. DOI: https://doi.org/10.1590/0034-71672017-0290.

9. García IAB. Dialéctica de la creación y la innovación en la filosofía de la praxis de Adolfo Sánchez Vázquez. RESF [Internet], 2017 [cited 2019 Jan 20]; 42(2):229-45. DOI: https://doi.org/10.5209/RESF.57336

10. Winters JRF, Do Prado ML, Heidemann ITSB. Nursing education oriented to the principles of the Unified Health System: perception of graduates. Esc. Anna Nery [Internet], 2016 [cited 2019 Jan 20]; 20(2):248-53. Available from: https://www.scielo.br/scielo.php?pid=S1414$81452016000200248 \&$ script=sci_arttext\&ting=en.

11. Araujo RM de L, Rodrigues DS. Filosofia da práxis e didática da educação profissional. Campinas, SP: Autores Associados; 2011.

12. Vargens OMC, Silva ACV, Progianti JM. The contribution of nurse midwives to consolidating humanized childbirth in maternity hospitals in Rio de Janeiro-Brazil. Esc. Anna Nery [Internet], 2017 [cited 2019 Jan 20]; 21(1):e20170015. Available from: https://www.scielo.br/scielo.php?script=sci_abstract\&pid=\$1414-81452017000100215\&lng=en\&nrm=iso\&tlng=es.

13. Carvalho EMP, Göttems LBD, Pires MRGM. Adherence to best care practices in normal birth: construction and validation of an instrument. Rev. Esc. Enferm. USP [Internet], 2015 [cited 2019 Jan 20]; 49(6):890-8. DOI: https://doi.org/10.1590/S0080-623420150000600003.

14. Leite CM, Pinto ICM, Fagundes TLQ. Permanent education in health: reproduction or counter-hegemony? Trab. educ. saúde [Internet], 2020 [cited 2020 Jul 03]; 18(Suppl. 1): e0025082. DOI: https://doi.org/10.1590/1981-7746-sol00250.

15. Silva GF, Moura MAV, Almansa Martinez P, Souza IEO, Queiroz ABA, Pereira ALF. Training in the obstetric nursing residency modality: a hermeneutic-dialectic analysis. Esc. Anna Nery [Internet], 2020 [cited 2020 Jul 09]; 24(4):e20190387. DOI: https://doi.org/10.1590/21779465-EAN-2019-0387.

16. Leite JO, Arcoverde ACB. Hegemony and Philosophy of Praxis: the challenges to social service. Rev. katálysis [Internet], 2017 [cited 2020 Jul 20]; 20(1):37-46. DOI: https://dx.doi.org/10.1590/1414-49802017.00100005.

17. Sarges RC, López LC. The delivery care in view of obstetric nurses in a public hospital: demedicalization and micropolitics from the gender language. VRA [Internet], 2016 [cited 2019 Jan 20]; 1(48):133-48. Available from: https://periodicos.ufrn.br/vivencia/article/view/11574. 